\title{
Rheumatic Bicuspid Aortic Valve Causing Device Emboli- zation during Transcatheter Aortic Valve Replacement
}

\author{
Manshu Yan, Elyse Guran, Annie Wang, Uoo Kim* \\ Loma Linda University School of Medicine Department of Anaesthesiology, USA
}

\begin{abstract}
Transcatheter aortic valve replacement (TAVR) has emerged as a feasible alternative for treatment of severe aortic stenosis with comparable outcomes to surgical aortic valve replacement (SAVR) in recent years. We present a case of device embolization in the left ventricular outflow tract (LVOT) during TAVR in a patient with severe aortic stenosis that required emergent surgical intervention. During the open-heart surgery for embolized prosthesis extraction and SAVR, both TEE exam and surgical specimen demonstrated bicuspid aortic valve and rheumatic nature of the valve with lack of calcification, which were identified to be the two main factors that contributed to the complication. In which the insufficient annular calcification increases the risk of device embolization due to lack of an adequate landing zone for device anchoring, and the anatomy of bicuspid valve contributes to the complication due to its associated large annular size and horizontal aorta. This case highlights device embolization as one possible complication of TAVR which is associated with substantial morbidity and mortality, the clinical management process was thoroughly documented with aortic angiography and transoesophageal echocardiography imaging.
\end{abstract}

Keywords: Transcatheter aortic valve replacement, Device embolization, Rheumatic valve, Bicuspid aortic valve

\section{Introduction}

The role of transcatheter aortic valve replacement (TAVR) in the treatment of patients with severe aortic stenosis (AS) has evolved since its first FDA approval in 2012. The landmark series of Placement of Aortic Transcatheter Valve (PARTNER) trials reflected robust evidence for TAVR in high, intermediate, and low risk patients. Moreover, technologic improvements and procedure simplification have contributed to increased volume of TAVRs. Complications of TAVR include access site or aortic vascular injury such as dissection, stenosis, perforation, rupture, and aneurysm. Other procedure related complications are associated with device landing zone which include aortic annular rupture, left ventricular perforation, cardiac tamponade, and device embolization. Conduction disturbances, stroke, paravalvular leak, and coronary obstruction can also occur after TAVR. Around 1\% of patients undergoing TAVR require surgical bailout with the most common reasons being valve dislodgement (22\%), ventricular rupture (19.9\%) and rupture of the aortic valve (14.2\%). ${ }^{1}$ Valve embolization is an infrequent event with an incidence of $0.2 \%$ to $1.7 \%$ and accounts for about $45 \%$ of emergency cardiac surgery in patients treated with TAVR. ${ }^{1}$ We present the case of a patient undergoing TAVR complicated by device embolization in the left ventricular outflow tract (LVOT) requiring emergent surgical intervention.

\section{Case Presentation}

A 50-year-old male with a past medical history of essential hypertension, paroxysmal atrial fibrillation, and cerebral vascular disease presented to our institution with palpitations, substernal chest pain and worsening shortness of breath. He was diagnosed with severe AS and evaluated by cardiothoracic surgery for surgical aortic valve replacement (SAVR). His predicted operative morbidity or mortality of surgical aortic valve replacement based on the Society for Thoracic Surgeons database (STS score) was calculated to be $1.4 \%$ (low risk), however, he rejected surgical intervention thus TAVR was planned. A comprehensive workup was performed
Quick Response Code:

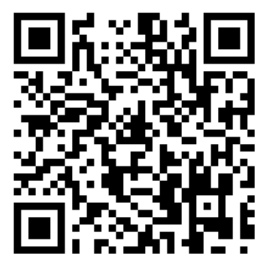

*Corresponding author: Uoo Kim, Assistant Professor, Anaesthesiology Associate Director, Liver Transplant Loma Linda University School of Medicine Department of Anaesthesiology, Division of Cardiothoracic Anaesthesiology, Loma Linda University Medical Centre, 11234 Anderson Street, MC-2532, Loma Linda, CA 92354, USA

Received: 14 February, 2021

Published: 23 February, 2021

Citation: Manshu Y, Elyse G, Annie W, Uoo K. Rheumatic Bicuspid Aortic Valve Causing Device Embolization during Transcatheter Aortic Valve Replacement. SOJ Cardio Curr Trend Surg. 2021;1(1):1-5. DOI: 10.53902/SOJCCTS.2021.01.000504 
including transthoracic echocardiography (TTE) which revealed a left ventricular ejection fraction (LVEF) of 35\%, mildly dilated ascending aorta, and severe aortic valve stenosis with the following measurements: LVOT $2.0 \mathrm{~cm}$, annulus $2.5 \mathrm{~cm}, \mathrm{~V} 1$ of $0.8, \mathrm{~V} 2 \mathrm{of} 3.9 \mathrm{~m} / \mathrm{s}$, MG $40 \mathrm{mmHg}$, AVA $0.8 \mathrm{~cm}^{2}$, and no aortic regurgitation (AR). However, the aortic valve morphology was unclear and appeared to be congenital bicuspid or unicuspid on TTE. Computed tomographic (CT) evaluation of the heart revealed a tricuspid aortic valve with a hypo plastic right cusp and severe aortic valve calcification. Coronary angiogram revealed normal coronary arteries. All studies were reviewed by the multidisciplinary heart team and the decision was made to proceed with transfemoral TAVR.

In the interventional cardiology suite, monitored anaesthesia care (MAC) with a remifentanil infusion ranging from 0.05 -
$0.07 \mathrm{mcg} / \mathrm{kg} / \mathrm{minute}$ was initiated according to our institutional protocol. Venous and arterial femoral access was obtained by the interventional cardiology team for intraoperative blood pressure monitoring and resuscitation. A temporary pacemaker was advanced through the venous sheath and positioned into the right ventricle. The optimal angle of deployment was determined with fluoroscopy. The native aortic valve was crossed with a guide wire and a balloon valvuloplasty was performed. Subsequently, the valve delivery system with a $26 \mathrm{~mm}$ Edwards SAPIEN3 valve was positioned across the aortic annulus and confirmed by aortic angiogram. The valve was then deployed under rapid ventricular pacing. During deployment, the delivery system including the valve slid along the native aortic valve, which resulted in device embolization in the left ventricular outflow tract (Figure 1).
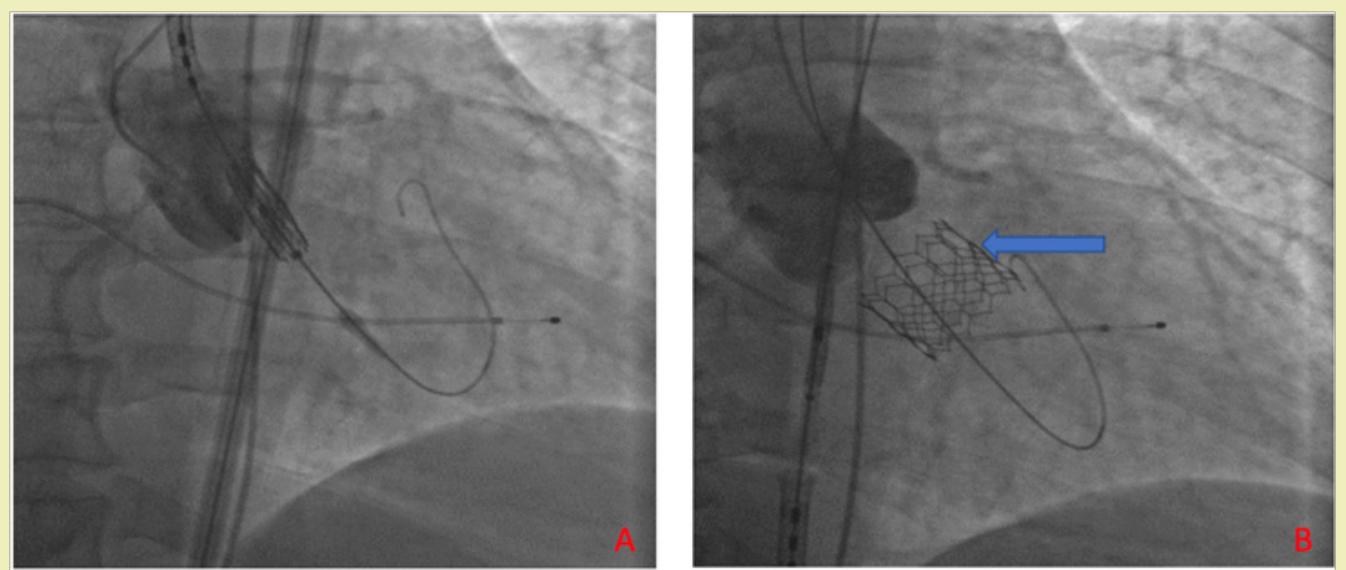

Figure 1: (A)Valve delivery system visualized across the aortic annulus with confirmation by aortic angiogram. (B) Embolization of the mechanical aortic valve device (arrow), visualized in the left ventricular outflow tract under aortic angiogram.



Figure 2: (A)Aortic valve long axis view, guidewire (orange arrow) passing through the native aortic valve, securing the embolized mechanical prosthesis (blue arrow). (B) Four chamber view, embolized mechanical prosthesis (arrow) visualized in the LVOT, between the anterior leaflet of the mitral valve and basal anteroseptal wall of the left ventricle. (C) modified two chamber view, embolized mechanicalprosthesis (arrow) visualized in the left ventricle with close proximity to the mitral leaflets. (D) 3D aortic valve long axis view, embolized mechanical prosthesis (arrow) visualized in the LVOT, caught between the anterior leaflet of the mitral valve and basal anteroseptal wall of the left ventricle. 
Subsequent angiography demonstrated intact flow through the device and the patient remained hemodynamically stable without vasopressors or inotropes. He was emergently transferred to the operating room (OR) for retrieval of the TAVR prosthesis and for SAVR. In the OR the patient was intubated, a left internal jugular central line was placed, and a TEE probe was inserted.

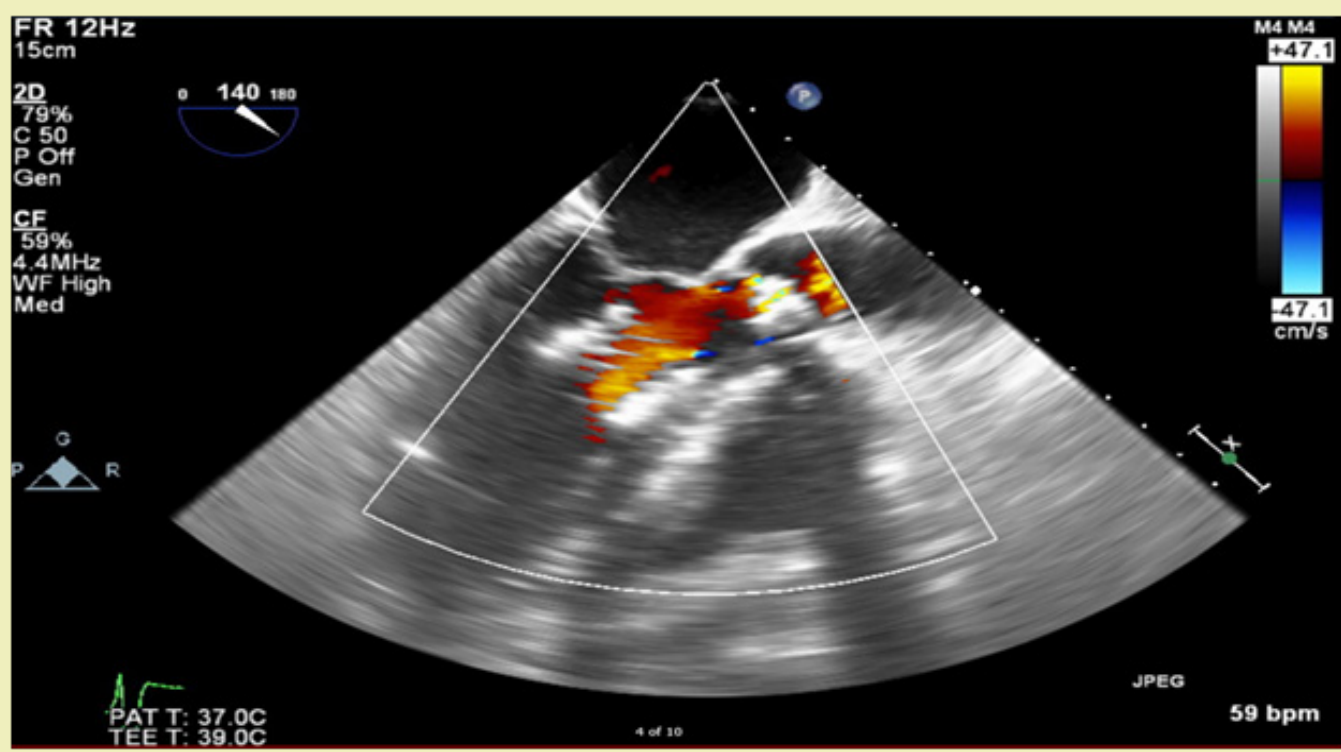

Figure 3: Flow through the embolized mechanical prosthesis (arrow) visualized under color flow Doppler via TEE.

On TEE examination, the Edwards SAPIEN3 valve was visualized in the LVOT, between the anterior leaflet of the mitral valve and anteroseptal wall of the left ventricle, and the mechanical prosthesis was held in place by a guide wire to ensure the device would not migrate distally or damage the ventricle by moving around freely
(Figure 2). There was adequate flow visualized through the LVOT without aortic or mitral valve regurgitation (Figure 3). And the native aortic valve was visualized as bicuspid and rheumatic in nature, with minimal calcifications on further TEE examination (Figure 4).

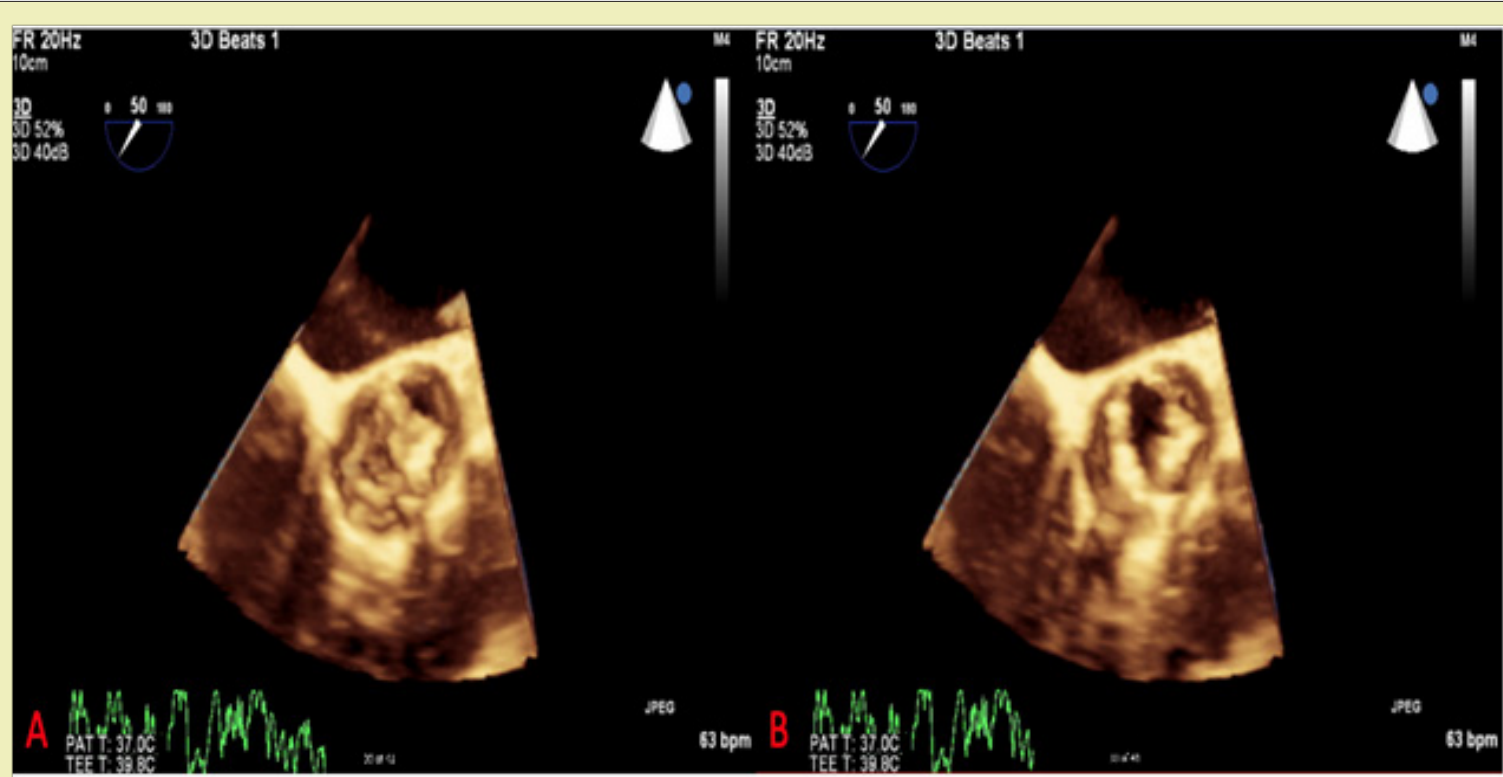

Figure 4: (A) Aortic valve short axis view visualizing the native bicuspid aortic valve during diastole. (B) Aortic valve short axis view visualizing the native bicuspid aortic valve during systole.

Surgery was initiated and the patient was placed on cardiopulmonary bypass (CPB). The surgical team then performed a transverse aortotomy. The native aortic valve was exposed and found to be congenitally bicuspid with minimal calcium on both the leaflets and annulus. In addition, the leaflets were rubbery and rheumatic in appearance. The native aortic valve was excised and the TAVR prosthesis was caught in the submitral apparatus, where it was untangled from the chordae, crushed, and withdrawn through the aorta (Figure 5). 


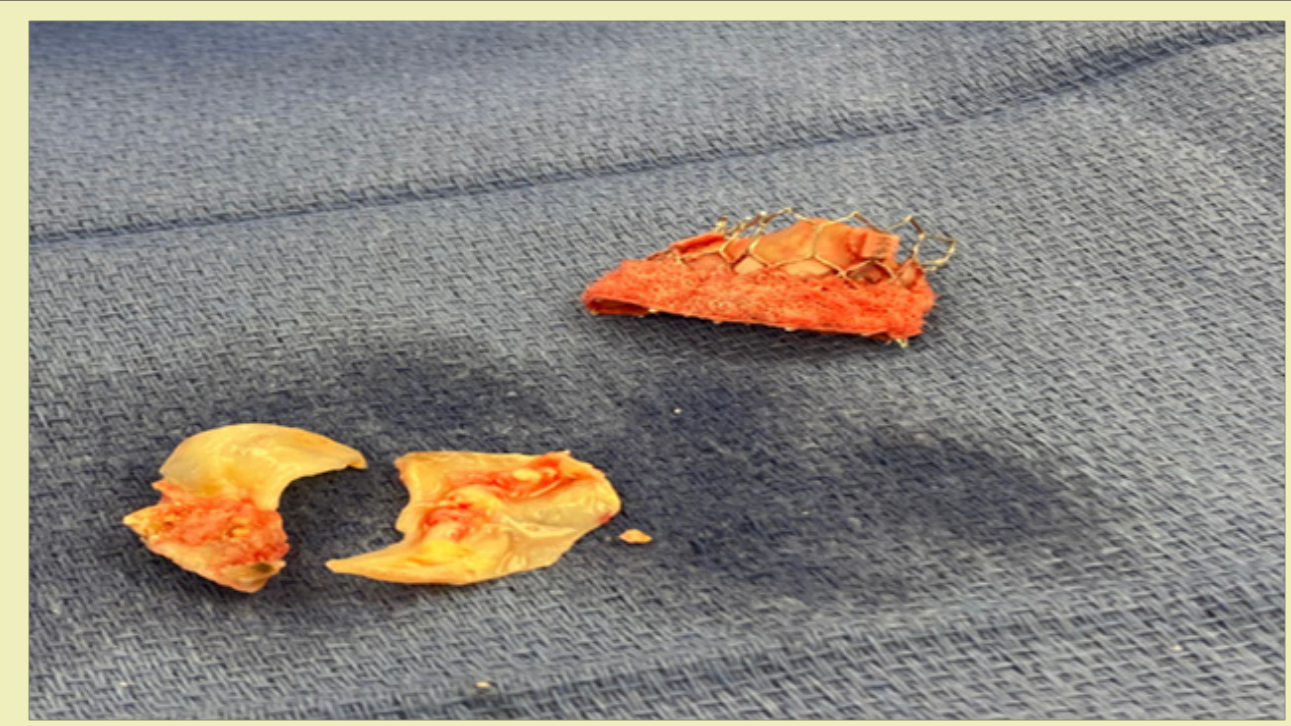

Figure 5: Extracted mechanical aortic valve prosthesis (blue arrow) and excised native bicuspid aortic valve (red arrow).

A $23 \mathrm{~mm}$ bioprosthetic valve was then implanted and a left atrial appendage ligation was performed. The patient was then rewarmed, ventilated and separated from CPB without difficulty on milrin one and low dose epinephrine infusions. The post CPB TEE demonstrated improved LVEF from 35\% to 50\%, no debris from the TAVR prosthesis or mitral valve damage were identified. The patient was transferred to cardiothoracic intensive care unit on a milrinone infusion, remained hemodynamically stable overnight and was extubated the next morning. He was discharged home on postoperative day 4 .

\section{Discussion}

Device embolization is an uncommon but potentially devastating complication of TAVR. Migration can occur in a cranial or caudal fashion resulting in aortic or LVOT translocation, respectively. ${ }^{1}$ Device embolization is associated with a 9-fold increase in mortality ${ }^{2}$ and approximately $45 \%$ of patients require urgent cardiac surgery. ${ }^{1}$ This case demonstrated one such complication with translocation into the LVOT requiring emergent open-heart surgery. Several factors contributed to this complication, primarily the lack of sufficient calcification on then ative valve as well as the patient's congenitally bicuspid aortic valve (BAV). The absence of annular calcification increases the risk of device embolization due to lack of an adequate landing zone for device anchoring. Though insufficient annular calcification likely played a major role, there have been studies examining TAVR in these populations. A recent meta-analysis examining TAVR for patients with native aortic valve regurgitation (NAVR), a population previously considered inappropriate for TAVR due to lack of adequate annular calcification, found that TAVR was a viable option for patients deemed too high risk forSAVR. ${ }^{3}$ Several additional anatomic and procedural factors have been cited as risks for device embolization. Anatomic factors include under sizing of the prosthesis, bulky calcified leaflet, horizontal annuli, and mitral prosthesis struts displacing the balloon. ${ }^{4}$ Procedural factors include poor coplanar angle, valve malposition, incomplete or delayed device balloon inflation, pacing failure, pre- mature pacing, post-dilatation implantation, stored wire tension, and failure to retract the transcatheter heart valve (THV) pusher. ${ }^{4}$ In a review of device embolization following TAVR, the most common causes identified were positioning errors followed by pacing errors. The same study showed a higher 30-day stroke and mortality rate associated with device embolization. ${ }^{5}$ Malposition of the valve usually occurs during placement ${ }^{5}$ but has also been seen up to twelve months after the procedure. ${ }^{4}$

The patient's previously unanticipated bicuspid aortic valve is another important factor to consider given the relative prevalence within the young adult and elderly population. Congenital BAV is the most common congenital cardiac abnormality and is the leading cause of AS in patients under age $65 .{ }^{6}$ It is estimated that $50 \%$ of adults with severe AS have a BAV. ${ }^{7}$ Bicuspid aortic valves become calcified with aging and can lead to aortic stenosis, aortic regurgitation, infective endocarditis, and even aortic dissection. ${ }^{7}$ Most patients with bicuspid aortic valve stenosis present at a younger age compared to tricuspid AS. ${ }^{6}$ For older patients with severe bicuspid AS, TAVR is a viable treatment due to increased risk of surgical complications from this cohort. However, the unique anatomy of the BAV can make the TAVR procedure more challenging due to its associated large annular size, asymmetric leaflet calcifications, ascending aortic dilatation, and horizontal aorta. ${ }^{8}$ TAVR for BAV stenosis was initially a contraindication due to the discontinuity between the circular bioprosthetic valve with the asymmetric annular BAV. Studies using new devices for bicuspid valves have shown promise with improved outcomes compared to first generation devices. ${ }^{9}$ SAPIEN 3, Lotus, and Evolut $\mathrm{R}$ valves have all been shown to be viable options for BAV with significant improvement in levels of paravalvular leak compared to first-generation valves. ${ }^{9}$ Interestingly, one multi-center study examining BAV TAVR with the SAPIEN 3 valve showed no cases of valve embolization or need for a second valve. ${ }^{10}$ The procedural and post procedural outcome were comparable using the newer generation device for BAV versus TAV, though the device success was slightly lower in the bicuspid AV 
group. ${ }^{11}$ However, a larger RCT trial is needed to better define the role of TAVR in BAV.12

The morphologic differences and subsequent challenges to successful TAVR in patients with BAV compared to tricuspid aortic stenosis make sufficient procedural planning essential. Although diagnosis of BAV with Doppler echocardiography is highly reliable with a predictive accuracy of $93 \%$, factors such as severe fibrosis, calcification, prominent raphe, and unclear coaptation line can lead to misinterpretation and misdiagnosis. ${ }^{13,14}$ Misclassification of BAV can be as high as $40 \%$ due to age, extent of calcification and image quality. ${ }^{14}$ Hence, accurate assessment of the aortic valve anatomy with multiple imaging modalities prior to proceeding with TAVR is critical. Morphology of the aortic valve is typically evaluated with TTE or TEE, with TEE providing higher accuracy in differentiating between bicuspid and tricuspid AV. ${ }^{13}$ Diagnosis is most reliable when two cusps are visualized during systole, specifically with the identification of two commissures forming an elliptical systolic orifice. ${ }^{15}$ TEE is also useful in further differentiating between rheumatic and calcific aortic stenosis, with rheumatic AS resulting in commissural fusion and a triangular systolic orifice. ${ }^{15}$ Whenever assessment of AV morphology is unclear with TTE, additional imaging such as TEE, magnetic resonance imaging (MRI), and CT should be considered. For this patient, preoperative workup with TTE revealed unclear aortic valve morphology, with concern for possible congenital bicuspid or unicuspid valve. However, CT revealed a tricuspid aortic valve with dysplastic or hypoplastic right cusp and severe aortic valve calcification. With two contradicting diagnoses, additional imaging with preoperative TEE could have been performed. Having had adequate information regarding the patient's specific valve morphology may have changed the procedural approach, device choice or even possibly the recommendation to proceed to SAVR instead of TAVR.

\section{Conclusion}

We present a case of device embolization during TAVR in a patient with severe aortic stenosis. In recent years TAVR has emerged as a feasible alternative for treatment of severe aortic stenosis with comparable outcomes to surgical replacement. This case highlights device embolization as one possible complication which is associated with substantial morbidity and mortality. Two main factors that contributed to this complication were lack of annular calcification and bicuspid morphology of the native aortic valve.

\section{Disclaimers}

This research did not receive any specific grant from funding agencies in the public, commercial, or not-for-profit sectors.

\section{Acknowledgments}

None.

\section{Funding}

None.

\section{Conflicts of interest}

Author declares that there is no conflict of interest.

\section{References}

1. Scarsini R, De Maria GL, Joseph J, et al. Impact of Complications During Transfemoral Transcatheter Aortic Valve Replacement: How Can They Be Avoided and Managed? J Am Heart Assoc. 2019;8(18):e013801.

2. Eggebrecht $\mathrm{H}$, Schmermund A, Kahlert P, et al. Emergent cardiac surgery during transcatheter aortic valve implantation (TAVI): a weighted meta-analysis of 9,251 patients from 46 studies. Euro Intervention. 2013;8(9):1072-1080.

3. Haddad A, Arwani R, Altayar O, et al. Transcatheter aortic valve replacement in patients with pure native aortic valve regurgitation: A systematic review and meta-analysis. Clin Cardiol. 2019;42(1):159-166.

4. Alkhouli M, Sievert H, Rihal CS. Device Embolization in Structural Heart Interventions: Incidence, Outcomes, and Retrieval Techniques. JACC Cardiovasc Interv. 2019;12(2):113-126.

5. Ibebuogu UN, Giri S, Bolorunduro 0, et al. Review of reported causes of device embolization following trans-catheter aortic valve implantation. Am J Cardiol. 2015;115(12):1767-1772.

6. Fedak PW, Verma S, David TE, et al. Clinical and pathophysiological implications of a bicuspid aortic valve. Circulation. 2002;106(8):900904.

7. Ward C. Clinical significance of the bicuspid aortic valve. Heart. 2000;83(1):81-85.

8. Das R, Puri R. Transcatheter treatment of bicuspid aortic valve disease: imaging and interventional considerations. Front Cardiovasc Med. 2018;5:91.

9. Alfadhli J, Jeraq $M$, Singh $V$, et al. Updates on transcatheter aortic valve replacement: Techniques, complications, outcome, and prognosis. $J$ Saudi Heart Assoc. 2018;30(4):340-348.

10. Perlman GY, Blanke P, Dvir D, et al. Bicuspid Aortic Valve Stenosis: Favorable Early Outcomes With a Next-Generation Transcatheter Heart Valve in a Multicenter Study. JACC Cardiovasc Interv. 2016;9(8):817-824.

11. Halim SA, Edwards FH, Dai D, et al. Outcomes of Transcatheter Aortic Valve Replacement in Patients With Bicuspid Aortic Valve Disease: A Report From the Society of Thoracic Surgeons/American College of Cardiology Transcatheter Valve Therapy Registry. Circulation. 2020;141(13):1071-1079.

12. Colombo A, Mangieri A. Surgery Versus TAVR for Bicuspid Aortic Valve Disease: The Time Has Come for a Randomized Study. JACC Cardiovasc Interv. 2020;13(9):1028-1029.

13. Brandenburg RO, Tajik AJ, Edwards WD, et al. Accuracy of 2-dimensional echocardiographic diagnosis of congenitally bicuspid aortic valve: echocardiographic-anatomic correlation in 115 patients. Am J Cardiol. 1983;51(9):1469-1473.

14. Cramer PM, Prakash SK. Misclassification of bicuspid aortic valves is common and varies by imaging modality and patient characteristics. Echocardiography. 2019;36(4);761-765.

15. Baumgartner H, Hung J, Bermejo J, et al. American Society of Echocardiography; European Association of Echocardiography. Echocardiographic assessment of valve stenosis: EAE/ASE recommendations for clinical practice. $\mathrm{J} A m$ Soc Echocardiogr. 2009;22(1):1-23. 